\title{
421 - Mood disorders and its relationship with cognitive impairment and dementia
}

AUTHORS: Sandra Torres (Psychiatry Resident in Centro-Hospitalar Barreiro-Montijo, Portugal), Andreia Lopes (Psychiatry Senior in Centro-Hospitalar Barreiro-Montijo, Portugal)

KEY-WORDS: mood disorders, cognitive impairment, dementia

\section{ABSTRACT:}

Mood disorders are common psychiatric illnesses that represent a major cause of disability worldwide. With life expectancy and the percentage of elderly people rising in many developed and undeveloped countries around the globe, cognitive impairment and dementia are gaining a societal importance. The relation between mood disorders and cognitive function is a twofold. On the one hand, cognitive deficits within mood disorders have been studied extensively, in which there seems to be a persistent neurocognitive impairment, both in acute phases and in interepisodic euthymic phases. Although results have not always been consistent, an overall pattern of specific impairments - in executive function, attention and memory - has become evident. On the other hand, recent research suggests that mood disorders, in general, may be risk factors for the development of mild cognitive impairment and dementia. In this sense, of the many models for the association of mood disorders and dementia, two are favored by several authors. One suggests that mood disorders are a risk factor for earlier clinical manifestation of dementia. The second sees mood disorders as the cause of dementing states, for instance through neuronal loss via dysregulation of the glucocorticoid cascade. In fact, there is suggestion that impairment of neuroplasticity may underlie the pathophysiology of mood disorders as such, and not only of neurocognitive impairment. In some patients, specific neurocognitive functions may be present before the onset of mood disorder and may constitute a trait factor or even an endophenotype. The aim of the present work is to, through a basic narrative review of published research on the main databases, summarize the main evidences of the association of mood disorders and dementia. 